\title{
ARTICLE
}

\section{Estimation for the intensity of beam loss by the optical fiber based Cherenkov beam loss monitor at SACLA}

\author{
Toshiro Itoga $\mathrm{a}^{\mathrm{a}^{*}}$ and Yoshihiro Asano \\ a Japan Synchrotron Radiation Research Institute,1-1-1 Koto, Sayo-cho, Sayo-gun, Hyogo-ken, 679-5198, Japan; \\ ${ }^{b}$ RIKEN, 1-1-1 Koto, Sayo-cho, Sayo-gun, Hyogo-ken, 670-5198, Japan
}

\begin{abstract}
An optical fiber based beam loss monitor has been installed and successfully working at an X-ray free-electron laser facility. This monitor gives the information about the position and the intensity of the beam loss over $140 \mathrm{~m}$ in real time. In this paper, calibration methods of a monitor signal with the thermo luminescent dosimeters and the simulation results were described.
\end{abstract}

Keywords: beam loss monitor; optical fiber; Cherenkov radiation; high energy electron; X-ray free-electron laser; Monte Carlo

\section{Introduction}

From the issues of radiation safety and radiation damage of accelerator equipments, it is useful to detect the beam loss immediately at high-energy and high intensity particle accelerator facilities.

In the undulator section of Spring-8 Angstrom Compact free electron LAser (SACLA) [1], beam loss monitors have been installed in order to control beam losses and to prevent demagnetization of permanent magnets due to electron hitting [2]. Since the commissioning phase of SACLA, this monitor has been successfully working.

This beam loss monitor consists of a quartz fiber with $400 \mu \mathrm{m}$ core diameter and a photomultiplier tube (PMT) which is connected with the upstream end of the fiber. The Cherenkov radiation which is produced in the fiber due to the passing of secondary electrons and positrons (Cherenkov emitters) is detected by the photomultiplier tube. With the detection time of the monitor signals, the position of the beam loss point can be measured. In addition, the use of two monitors with different gains allows measuring intensity of the beam loss over the wide dynamic range.

While it is expected that the waveform of the monitor signal corresponds to the spatial distribution of the electron flux or absorbed dose due to the beam loss, if this monitor is used for the beam loss control, it is necessary to calibrate the magnitude of the monitor signal to a value such as the number of electrons or the absorbed dose in order to set an appropriate threshold level.

In this study, the electron beam loss was measured by the beam loss monitor and thermo luminescent dosimeters (TLD), and the correlation among the signal of the beam loss monitor, the absorbed dose and electron flux was investigated.

\section{Experimental procedure}

Figure 1 shows the undulator section of SACLA. The 18 units of the in-vacuum undulators [3] and 5 units of the beam profile monitor systems [4] have been installed

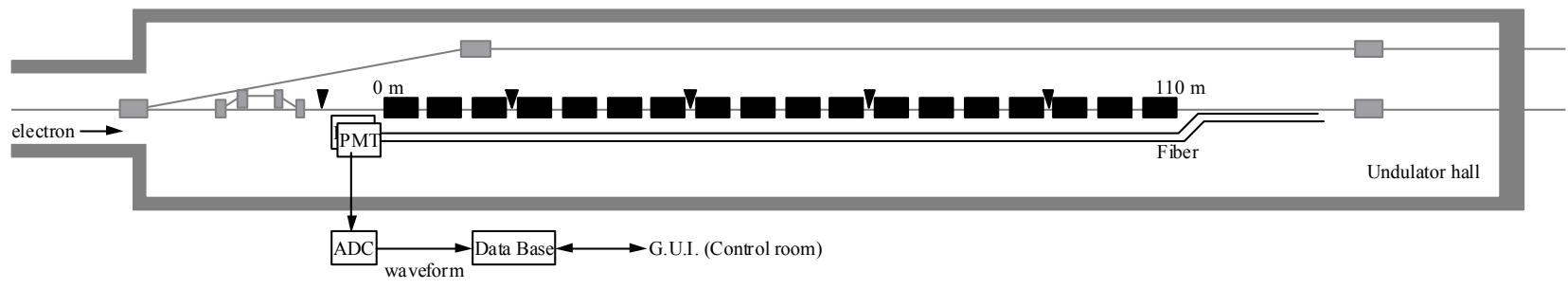

Figure 1. Schematic views of the undulator hall and the beam loss monitoring system. Black boxes and black triangles indicate the undulators and the positions of the beam profile monitors, respectively.

*Corresponding author. Email: itoga@spring8.or.jp 
in this section. The pair of optical fibers of the beam loss monitors has been installed along with the vacuum chamber all over the undulator section, as shown by the black solid line in the figure. The distance between the beam loss monitor and the electron beam path is $12.5 \mathrm{~cm}$ in the region of the undulator and $1.25 \mathrm{~cm}$ in the region of the simple vacuum pipe, respectively. The PMTs were set at the upstream end of the fibers in order to realize higher position resolution.

The TLDs made of LiF:Mg,Ti (TLD-100) have been set along the optical fiber around the upstream end and center of each undulator during this experiment, and these TLDs were calibrated to the absorbed dose in air by gamma-rays from a ${ }^{60} \mathrm{Co}$ source.

Beam losses occurred by using the screens of the beam profile monitors consisting of a Ce:YAG scintillator and a stainless steel mirror during the experiments. Both thicknesses are $100 \mu \mathrm{m}$.

The electron beam energy was $7.58 \mathrm{GeV}$ which was obtained from the wave length of the X-ray free-electron laser and the charge of each electron bunch was about $0.13 \mathrm{nC}$ which was measured by the current transformer [4]. The apertures of electron beam path in the undulators (the gap between the upper and lower magnet arrays) and in the vacuum pipes were $40 \mathrm{~mm}$ and $21 \mathrm{~mm}$, respectively.

\section{Simulation}

An estimation of the monitor output was performed using the Monte Carlo code FLUKA [5, 6]. The screens of the beam profile monitors, the vacuum chambers and the magnet arrays in the undulator have been reproduced in the geometry of the simulation. Only the electrons, positrons and gamma-rays which had the kinematic energy above $190 \mathrm{keV}$ were considered in the simulation.

The position, the direction and the kinematic energies of the Cherenkov emitters passing through the optical fibers were scored in the FLUKA simulation. In order to detect the Cherenkov radiation at the upstream end of the fiber, the events must satisfy the three conditions as follows. (1) The Cherenkov emitters scatter in the upstream direction. (2) Those kinematic energy are beyond the threshold energy in order to produce Cherenkov radiation (Eq. 1). (3) The Cherenkov angle is in the acceptance of the fiber (Eqs. 2 - 4).

$$
\begin{aligned}
& \frac{v}{c}>\frac{1}{n} \\
& \left|\theta_{c}-\theta_{A}\right| \leq \psi_{e} \leq\left|\theta_{c}+\theta_{A}\right| \\
& \theta_{c}=\cos ^{-1}(c / v n) \\
& \theta_{A}=\sin ^{-1}(N A)
\end{aligned}
$$

Where, $v$ is the velocity of the Cherenkov emitter, $c$ is the velocity of light, $n$ is the index of the fiber material, $N A$ is the numerical aperture of the fiber. $\Psi_{e}$ is the angle between the path of the Cherenkov emitter and the fiber, $\theta_{c}$ is the Cherenkov angle and $\theta_{A}$ is the acceptance angle of the fiber.

Finally, the path length of each Cherenkov emitter in the fiber was summed up, since the intensity of the Cherenkov radiation is proportional to the path length of the Cherenkov emitters in the fiber.

In addition, the spatial distribution of the absorbed dose by the electro-magnetic events at the position of the optical fiber was also calculated.

\section{Results and discussions}

Figure 2 shows the simulation results of Cherenkov intensity and the spatial distribution of the number of the electrons and positrons hitting the fiber. These are almost constant within a few times of magnitude at each position because of the small variance of the total path length in the fiber.

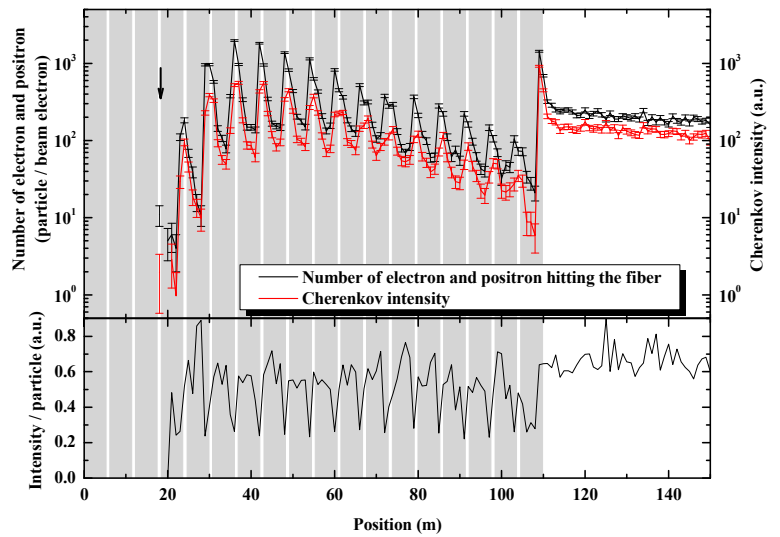

Figure 2. The simulation results of both the Cherenkov intensity (black line) and the number of electrons (positrons) hitting the fiber per one beam electron (red line) (upper). The ratio between the Cherenkov intensity and the number of electrons (lower). The profile monitor was inserted at $18 \mathrm{~m}$ (black arrow). The gray areas indicate the undulators. The errors bars indicate the standard deviation.

Figure 3 shows the an example of the measured beam loss spatial distributions by the beam loss monitor, together with the simulation results. The monitor signals from both high and low gain monitors were combined and compensated for the light attenuation in the fiber.

Most of the scattered electrons by the screen were lost between the undulators, since the aperture was small. A large loss seemed to occurre in the downstream region of the undulator section. It was caused by the small aperture and short distance between the fiber and the beam path. One of the causes of a discrepancy between measurement and simulation in the downstream region may be attributed to the lack of massive components such as a yoke of quadrupole magnet in the simulation.

Although the relation between the Cherenkov 
intensity and the electron (positron) flux in the fiber is expected to be almost constant from Figure 2 (lower), there is the discrepancy between Cherenkov intensity obtained by the beam loss monitor and the simulation as shown in Figure 3 (lower). Further investigation is necessary to calibrate the monitor signal with the electron flux.

Figure 4 shows the cumulative dose distribution obtained by using the TLDs and the simulation with the signal of the beam loss monitor. These data were integrated during the experiment $(90$ minutes in $1 \mathrm{~Hz}$ operation). Beam losses occurred by using the 5 screens.

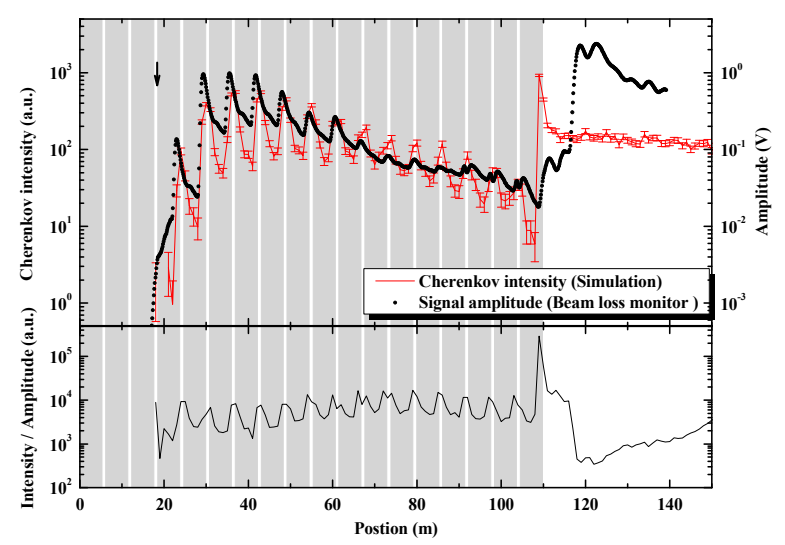

Figure 3. The beam loss spatial distribution (upper) measured by the beam loss monitor (average of 400 bunches) (solid circles) with the simulated Cherenkov intensity (red line). The ratio between the experimental data (beam loss monitor) and the simulation result (lower). The standard deviations of the experimental data were typically less than $10 \%$.

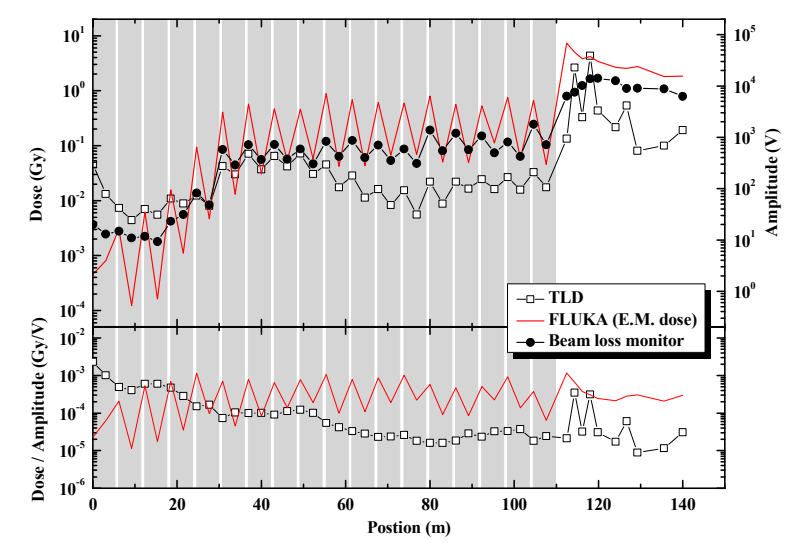

Figure 4. The absorbed dose spatial distributions obtained by both the TLDs (open square) and the simulation (red line) with the signal of the beam loss monitor (upper). These results have been integrated during the experiment ( 90 minutes, $1 \mathrm{~Hz}$ operation). The ratio between the absorbed dose and the signal of the beam loss monitor (lower). Almost all the standard deviations of these values are less than $10 \%$.

The results of the simulation and the beam loss monitor have roughly similar distribution. However, the simulation results of the absorbed dose are higher than the experimental results by using the TLD in almost the whole region. The reason of this discrepancy is that the TLDs are expected to underestimate the absorbed dose due to the charged particles. The further investigations are required to clarify the reason of this discrepancy.

In the region from 60 to $100 \mathrm{~m}$, the ratios between the results obtained by the TLDs and the beam loss monitor agree in a few times of magnitude. On the other hand, the overall ratios between these spatial distributions change on a large scale. One of the causes of this discrepancy is that this monitor has no sensitivity to the electrons and the positrons with the energy below the threshold energy defined by the Eq. $1(190 \mathrm{keV}$ in this case).

\section{Conclusion}

Calibration studies of the optical fiber based Cherenkov beam loss monitor have been carried out with the TLD and the simulation results.

The all results of the experiment and the simulation show the roughly similar tendency with the monitor signals. However, the relations between these results and the monitor signals have large variance respecting to the position. The further investigations therefore are needed in order to use this monitor for the beam loss control as a subsidiary to a radiation control system.

\section{References}

[1] T. Ishikawa, H. Aoyagi, T. Asaka, Y. Asano, N. Azumi, T. Bizen, H. Ego, K. Fukami, T. Fukui, Y. Furukawa, S. Goto, H. Hanaki, T. Hara, T. Hasegawa, T. Hatsui, A. Higashiya, T. Hirono, N. Hosoda, M. Ishii, T. Inagaki, Y. Inubushi, T. Itoga, Y. Joti, M. Kago, T. Kameshima, H. Kimura, Y. Kirihara, A. Kiyomichi, T. Kobayashi, C. Kondo, T. Kudo, H. Maesaka, X. M. Marechal, T. Masuda, S. Matsubara, T. Matsumoto, T. Matsushita, S. Matsui, M. Nagasono, N. Nariyama, H. Ohashi, T. Ohata, T. Ohshima, S. Ono, Y. Otake, C. Saji, T. Sakurai, T. Sato, K. Sawada, T. Seike, K. Shirasawa, T. Sugimoto, S. Suzuki, S. Takahashi, H. Takebe, K. Takeshita, K. Tamasaku, H. Tanaka, R. Tanaka, T. Tanaka, T. Togashi, K. Togawa, A. Tokuhisa, H. Tomizawa, K. Tono, S. Wu, M. Yabashi, M. Yamaga, A. Yamashita, K. Yanagida, C. Zhang, T. Shintake, H. Kitamura and N. Kumagai, A compact X-ray free-electron laser emitting in the sub-ångström region, Nat. Photon. 6 (2012), pp. 540-544.

[2] X.-M. Marechal, Y. Asano and T. Itoga, Design, development, and operation of a fiber-based Cherenkov beam loss monitor at the SPring Angstrom Compact Free Electron Laser, Nucl. Instrum. and Meth. A 673 (2012), pp. 32-45.

[3] H. Kitamura, Recent trends of insertion-device technology for X-ray sources, J. Synchrotron Rad. 7 (2000), pp. 121-130.

[4] Y. Otake, H. Maesaka, S. Matsubara, C. Kondo, T. 
Sakurai, K. Yanagida, H. Ego, T. Matsumoto, H. Tomizawa and $\mathrm{S}$. Inoue, Commissioning and performance of the beam monitor system for XFEL/SPRING-8 "SACLA", Proc. of IPAC2011, San Sebastian, Spain, Sept. 4-9, 2011, (2011), MOOCB02.

[5] G. Battistoni, S. Muraro, P.R. Sala, F. Cerutti, A. Ferrari, S. Roesler, A. Fasso and J. Ranft, The
FLUKA code: Description and benchmarking, AIP Conf. Proc. 896, Batavia, USA, Sept. 6-8, 2006, (2007), pp. 31-49.

[6] A. Ferrari, P.R. Sala, A. Fasso and J. Ranft, FLUKA a Multi-particle Transport Code, CERN-2005-10 (2005), INFN/TC_05/11, SLAC-R-773. 